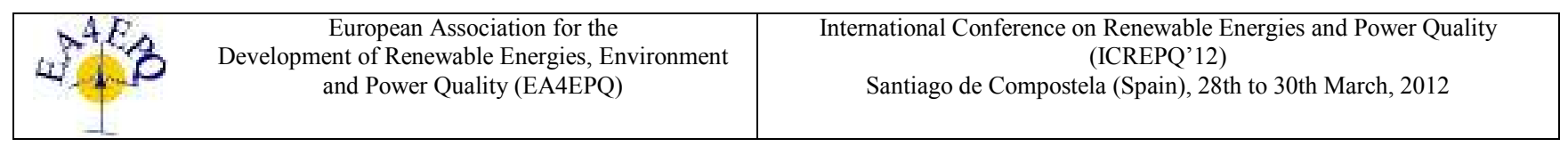

\title{
Pressurized concentrated solar power receiver designed to operate with closed Brayton cycles
}

\author{
R. Ferreiro Garcia ${ }^{1}$, R. Borras Formoso ${ }^{1}$, A. DeMiguel Catoira ${ }^{2}$, and J. Romero Gomez ${ }^{2}$ \\ ${ }^{1}$ Department of Industrial Engineering \\ E.T.S.N.M., A Coruna University \\ Campus Riazor, 15011 A Coruna (Spain) \\ Phone/Fax number:+0034 981 167000: 4205 e-mail: ferreiro@udc.es;
}

${ }^{2}$ Departmenrt of Energy and Propulsion

E.T.S.N.M., A Coruna University

Campus Riazor, 15011 A Coruna (Spain)

Phone/Fax number:+0034 981 167000: 4205

\begin{abstract}
Parabolic through and parabolic dish concentrators (PDC) followed by a volumetric receiver is proposed, studying the performance behavior of a closed Brayton cycle (CBC) operating with $\mathrm{He}$ and N2. A pressurized gas (helium, nitrogen) circulates along the volumetric receiver capturing the concentrated thermal solar energy which is converted into mechanical energy by a CBC. The overall efficiency of the plant (PC-Receiver $+\mathrm{CBC}$ ) is being computed under variable parameters to determine the operating conditions for which efficiency is acceptable. As consequence of the proposed analysis, it is concluded that direct coupling between volumetric receiver and thermal engine renders high efficiency while avoiding a heat exchanger.
\end{abstract}

\section{Key words}

Concentrated solar power, Parabolic dish, Parabolic through, Volumetric receiver, Thermal efficiency, Working fluid.

\section{Introduction}

Concentrated solar energy is an option that clearly contributes to increase the energy density of the solar radiation, which conducts us to the possibility of using absorbers with small surfaces. Since the objective is to convert the concentrated solar energy into useful work, the theory and achieved experience suggests that it can be done more efficiently the higher the temperature is.

The objective of the study is to capture solar radiation by means of a novel pressurized volumetric receiver and convert it into electric power via a closed Brayton cycle. The capture of solar radiation and conversion of it into solar thermal energy involves the use of solar receivers. In order to improve these solar receivers, some studies have been carried out using computer models and experimental data [1], [2], [3]. A lot of research has been carried out on designing new receivers or improving the performance of existing ones $[4 ; 5]$, and on understanding factors that affect their performance [6]; [7]. One important group of mentioned receivers is the volumetric solar receivers.

Volumetric receivers are generally used in central receiver solar power plants and have also been used for chemical applications [2] where the applied heat transfer fluid (HTF) is usually air or any other suitable gas such as helium or hydrogen. Several studies have been carried out on volumetric solar receivers that use air as a HTF [2]; [8;] [9]. In order to increase the heat transfer surfaces and consequently the transfer efficiency, the use of wire mesh, ceramic absorbers and other porous materials in these receivers has been applied with the aim of increase their efficiency [3]; [9]; [10]; [11]; [12]; [13], [14].

The reviewed references don't indicate a large use of volumetric receivers applied on indirect solar heat exchangers that have a separate solar collector followed by a power unit or thermal energy storage system [15], [16], [17]. Moreover, these receivers have mainly been used with air as a HTF and metal foams as the porous media. Furthermore, most of the studies on metal foams have focussed on their pressure drop and flow characteristics. Among the studies that have focused on the thermal conductivity of these metals foams, very few have dealt with their characteristics under concentrated solar radiation (CSR) and using a liquid as the HTF. In this study, heat transfer gas is used as the HTF and a wire mesh is used to improve the effective thermal conductivity and the flow of the gas in the proposed receiver. The wire mesh was chosen as the candidate for investigation because it is cheap and readily available.

\section{Description of the high temperature receiver}


The design consists of a metal receiver with conical and pyramidal shapes depending on the concentrator type (parabolic dish or parabolic through) equipped with input and output pipes to permit the gas circulation. The shapes of the receivers, the surface shapes and the dimensions of the receiver apertures were chosen based on the work referenced in [16]. Fig. 1 shows the distribution of the light rays on this receiver equipped with a thermal to electric energy convertor scheme, while in figure 2 the receiver structure is depicted showing the main components.

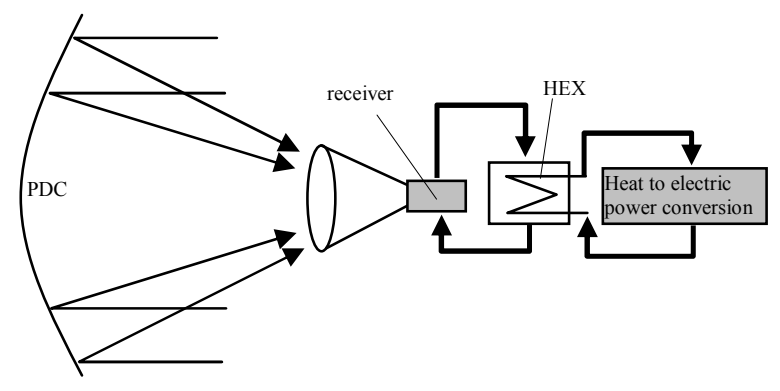

Fig. 1. Solar based power plant structure

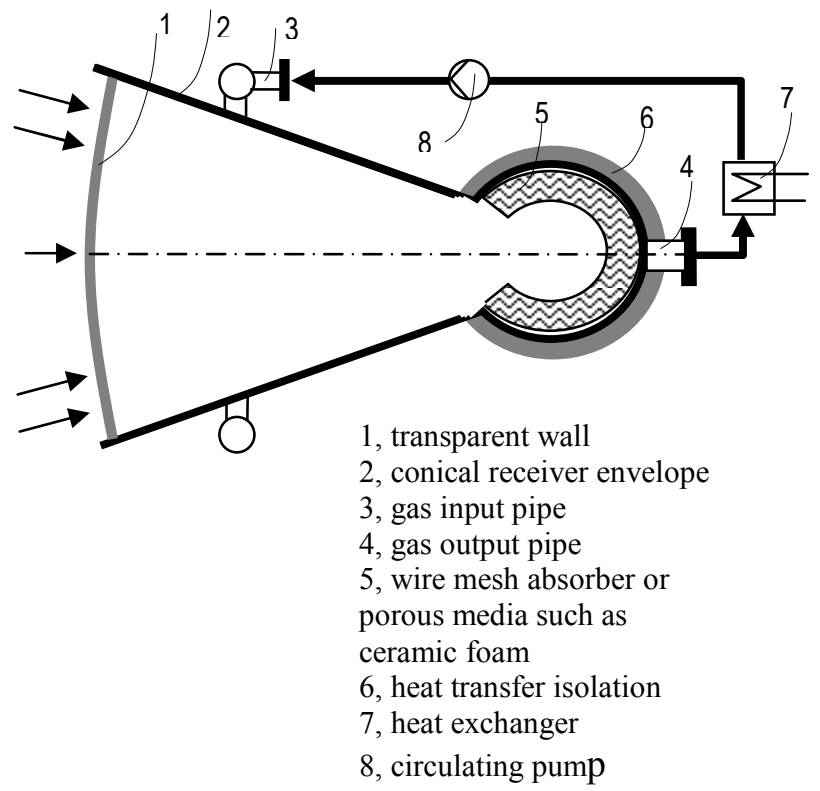

Fig. 2. The receiver cross-section configuration.

\section{A. Solar radiation to heat based power conversion}

The device that is used in high temperature solar concentrators for the conversion of concentrated solar radiation to heat is a receiver. It is designed to absorb the concentrated solar radiation and to transfer as much energy as possible to a HTF. Inherent unavoidable heat losses exists from the fact that the absorbing surface cannot be completely black, that it emits thermal radiation to the environment, because it has an elevated temperature, and that convection as well as conduction occur. Assuming that the receiver is affected by inherent heat losses (irreversibilities) the useful heat collected by the receiver can be estimated as

$$
Q_{U}=\dot{m} \cdot C_{P} \cdot(T o-T i)
$$

where

$\dot{m} \quad$ is the fluid mass flow rate

$C p \quad$ is the fluid specific heat capacity

To is the fluid output temperature from the receiver

$\mathrm{Ti}$ is the fluid input temperature to the receiver

The incident solar energy on the PDC is defined as

$$
Q s=A_{A p} \cdot C \cdot E_{S}
$$

where

$A_{A p} \quad$ is the PDC normal area

$E_{S} \quad$ is the direct solar radiation density sometimes assumed as $800 \mathrm{~W} / \mathrm{m}^{2}$, although a normal radiation density (a sun) is considered as $1000 \mathrm{~W} / \mathrm{m}^{2}$.

The thermal efficiency $\eta t h$ of the solar energy to heat power conversion composed by the PDC and receiver is defined by the ratio of the useful heat at the heat exchanger 7 to the incoming solar radiation on the PDC.

$$
\eta_{t h}=\frac{Q_{U}}{Q_{S}}=\frac{\dot{m} \cdot C_{P} \cdot(T o-T i)}{A_{A p} \cdot C \cdot E_{S}}
$$

The real scenario efficiency depends strongly on the receiver structure in such a way that a lot of concentrating process variables is involved. Taking into consideration experimental data with regard to the solar to heat conversion process it must be taken into account that

- higher fluid temperatures yields lower efficiencies of the PDC-receiver convertor.

- higher concentration ratios lead to higher efficiencies. -convection and conduction losses are of minor importance at high concentration ratios.

Generally speaking, for ideal thermodynamic cycles according Carnot, the thermal efficiency depends on the difference between high and low temperatures (high temperature at heat source and low temperature at heat sink). Since the objective is to achieve the highest technically possible overall efficiency, a compromise between both efficiencies must exist.

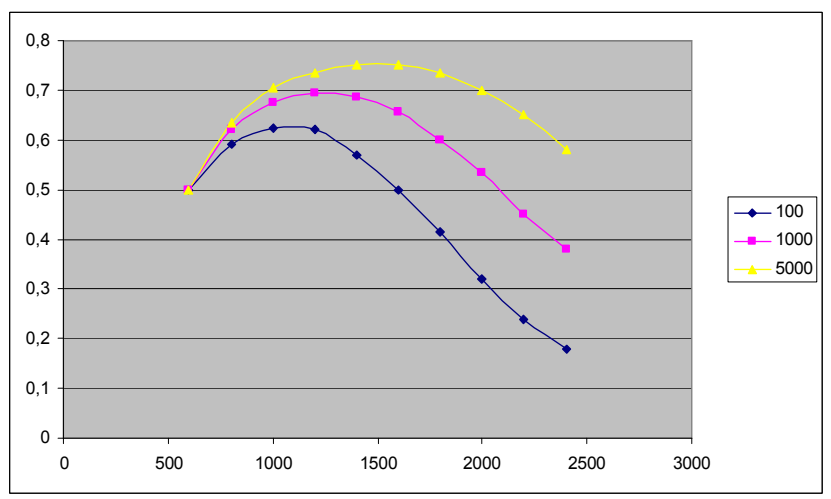

Fig. 3. The ideal total efficiency of a receiver corresponding to a high temperature solar concentrating system.

In figure 3 it is shown the total efficiency of a high temperature solar concentrating system for the generation of mechanical work as the function of the upper receiver temperature for different concentration ratios and a selective absorber, [17]. According this figure, the total efficiency of a generic volumetric receiver depends on a 
number of variables different of that mentioned. As consequence, the optimal efficiency deals with such variables more than with the top and down temperatures of the receiver.

\section{The Closed Brayton cycle based on the pressurized volumetric receiver.}

There are four processes in the basic CRC, each changing the state of the working fluid. These states are identified by state symbols (a, b, c and d) in the diagram shown in figure 4.

The ideal $\mathrm{RC}$ is inspired by the Carnot cycle. In figure 4 (a) it is depicted in the T-S diagram associated with the physical components shown in figure 4(b).

The thermodynamic efficiency of the cycle is defined as the ratio of net power output to heat input as shown in equation (4).

$\eta_{R}=\frac{W_{i}-W_{o}}{Q_{i}} \approx \frac{W_{n}}{Q_{i}}=\frac{Q_{i}-Q_{o}}{Q_{i}}$

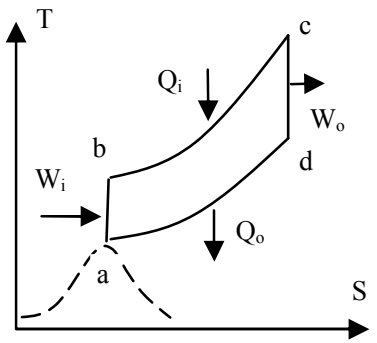

(a)

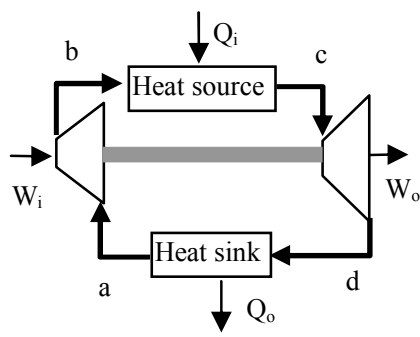

(b)
Fig. 4. Basic CBC: (a) the T-S diagram. (b), the basic plant structure

The general layout of a parabolic dish engine, receiver and power converter composed by $\mathrm{CBC}$ is shown in figure 5 . According such scheme, the structure of the power convertor consists of concentrated solar gas receiver heater which supplies pressurized gas to a gas turbine, which converts solar thermal energy into mechanical-electric energy, returning to the receiver heater impelled by a gas compressor.

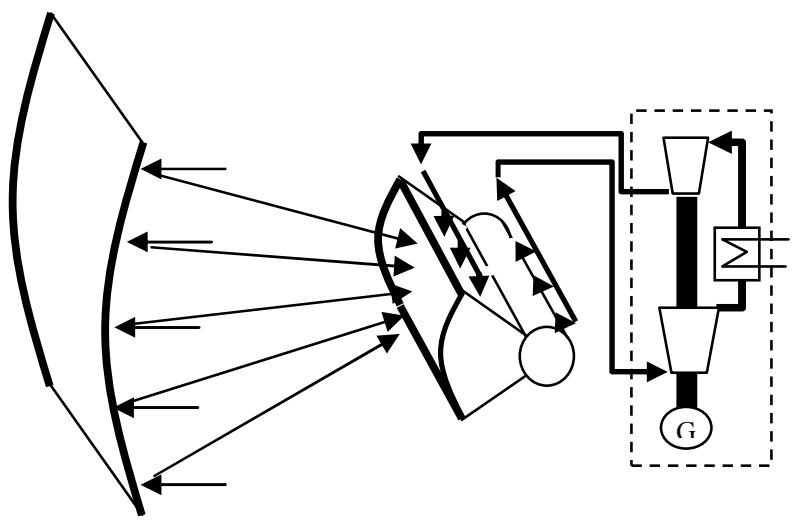

Fig. 5. The PDC coupled to a power generator based on the Rankine cycle.
The CBC structure shown in figure 5 has the advantage of direct transferring of solar energy from the volumetric receiver to the gas turbine, which implies to do without a complementary heat exchanger and consequently to increase the thermal efficiency of the cycle.

\section{The analysis of proposed $\mathrm{CBC}$ with a pressurized volumetric receiver.}

In the proposed analysis, an isentropic efficiency for $\mathrm{CBC}$ compressor and turbines is considered, which approaches the values of 0,85 and 0,90 respectively.

The main results of the proposed thermodynamic cycles for the mentioned working fluids are depicted in table 1, 2 and 3 respectively, where thermal cycle efficiency as function of top cycle temperatures and pressure ratios is presented.

Table I. - Efficiency of the CBC as function of the top cycle temperature and pressure ratio for helium as working fluid.

\begin{tabular}{r}
\hline T/rp 1600 1300 1000 \\
$328,9227,8125,39$ \\
$537,3835,2929,73$ \\
$842,838,5524,74$ \\
$1044,2638,2419,00$
\end{tabular}

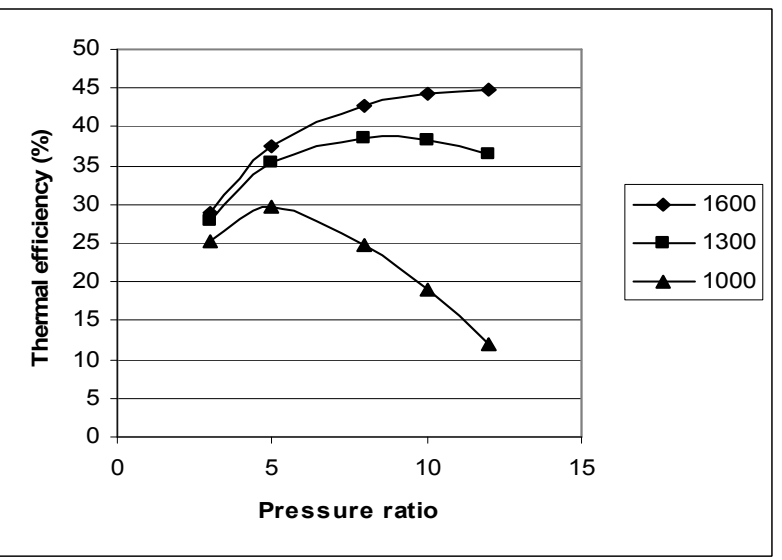

Fig. 6. Efficiency of the CBC as function of the top cycle temperature and pressure ratio for helium as working fluid

Table II. - Efficiency of the CBC as function of the top cycle temperature and pressure ratio for nitrogen as working fluid.

$\begin{array}{rrrr}\text { T/rp } & 1600 & 1300 & 1000 \\ 5 & 34,72 & 36,77 & 40,61 \\ 10 & 43,44 & 45,23 & 48,61 \\ 15 & 47,6 & 49,08 & 52,08 \\ 20 & 50,11 & 51,25 & 53,47 \\ 25 & 51,8 & 52,56 & 53,61 \\ 30 & 52,99 & 53,34 & 52,69 \\ 40 & 54,5 & & \end{array}$




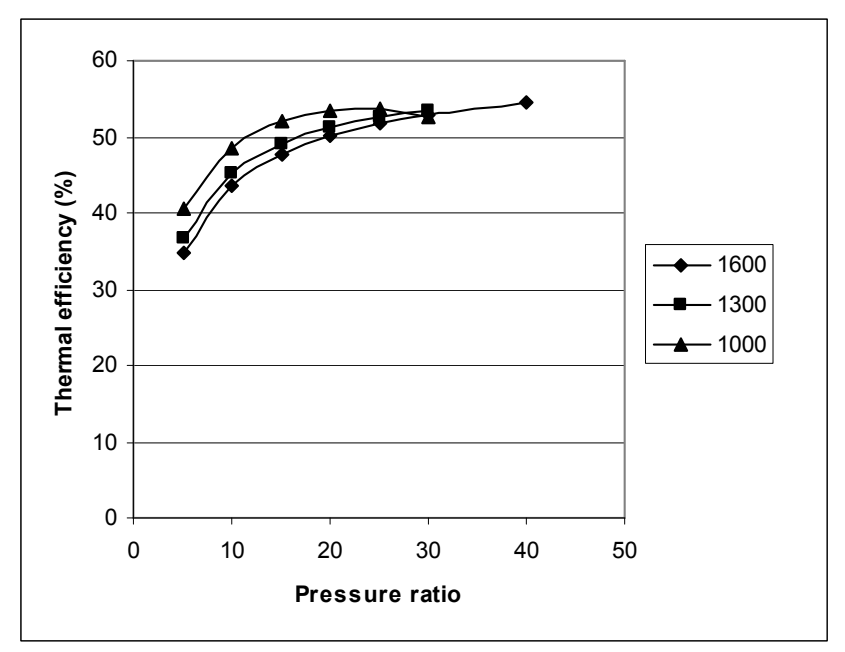

Fig. 7. Efficiency of the $\mathrm{CBC}$ as function of the top cycle temperature and pressure ratio for nitrogen working fluid.

The main results of the performed $\mathrm{CBC}$ analysis with regard to the cycle thermal efficiency for the mentioned working fluids depicted in tables 1 and 2 respectively, are shown in figures 6, and 7. Results show that the proposed volumetric receiver is suitable to supply thermal energy to $\mathrm{CBC}$ with high thermal efficiency.

\section{Conclusions}

The results shown in figures 3,6 and 7, as well as tables I and II, suggest to implement solar energy based volumetric receivers directly coupled to thermal engines. The behaviour of helium as working fluid is poor that that of nitrogen. The increase of thermal efficiency and simplification of installation equipment is a direct consequence of such structure. Another important consequence of such structure is that moderate receiver temperatures $(1000 \mathrm{~K})$ at relative low pressure ratios (10) can render high thermal efficiency when heat sink temperatures are set around $290 \mathrm{~K}$. Furthermore, nitrogen is significantly more efficient that helium at such low temperatures, although the specific work is lower.

\section{References}

[1] P.O Jarvinen, Heat mirrored solar energy receivers, Journal of Energy 2 (1978) 95-99.

[2] R. Pitz-Paal, B. Hoffschmidt, M. Böhmer, M. Becker, Experimental and Nummerical evaluation of the performance and flow stability of different types of open volumetric absorbers under non-homogeneous irradiation, Solar Energy 60 (1997) 135150.

[3] A. Kribus, P. Doron, R. Rubin, J. Karni, R. Reuven, S. Duchan, E. Taragan, A multistage solar receiver: The route to high temperature, Solar Energy 67 (1999) 3-11.

[4] A. Yogev, M. Wolfson, A window for central solar receiver with volumetric absorber, World Intellectual Property Organisation (1996), International Publication number WO 96/12918.

[5] R. Buck, C. Barth, M. Eck, W. Steinmann, Dual receiver concept for solar towers, Solar Energy 80 (2006) 1249-1254.

[6] A.M. Clausing, An analysis of convective loss from cavity solar central receivers, Solar Energy 27 (1981) 295-300.
[7] M. Prakash, S.B. Kedare, J.K. Nayak, Investigation on heat loss from a solar cavity receiver, Solar Energy 83 (2009) 157170.

[8] M. Becker, S. Cordes, M. Böhmer, The development of open volumetric receivers, Proceedings of the 6th symposium on Solar Thermal Concentrating Technologies, Sept-Oct, 1992, CIEMAT, ed., Madrid, Spain, II, 945-952.

[9] M. Böhmer, C. Chaza, The ceramic foil volumetric receiver, Solar Energy Materials 24 (1991) 182-191.

[10] P. Hellers, M. Pfänder, T. Denk, T. Felix, A. Valerde, J. Fernandez, A. Rind, Test and evaluation of a solar powered gas turbine system, Solar Energy 80 (2006) 1225-1230.

[11] P. Stobbe, B. Hoffshmidt, Hybrid volumetric receiver assembly and process for producing such assembly, World Intellectual Property Organisation (2003), International publication number WO 03/021160 A1.

[12] K. Boomsma, D. Poulikakos, F. Zwick, Metal foams as compact high performance heat exchangers, Mechanics of Materials 35 (2003) 1161-1176.

[13] T. Fend, R. Pitz-Paal, O. Reutter, J. Bauer, B. Hoffschmidt, Two novel highporosity materials as volumetric receivers for concentrated solar radiation, Solar Energy Materials \& Solar Cells 84 (2004) 291-304.

[14] Antonio L. Avila-Marín, Volumetric receivers in Solar Thermal Power Plants with Central Receiver System technology: A review, Solar Energy 85 (2011) 891-910.

[15] A. Mawire and M. McPherson, Experimental characterization of a thermal energy storage system using temperature and power controlled charging, Renewable Energy 33 (2008) 682-693.

[16] A. Mawire, M. McPherson, R.R.J. van den Heetkamp, S.J.P. Mlatho, Simulated performance of a storage materials for pebble bed thermal energy storage (TES) system, Applied Energy 86 (2009)1246-1252.

[17] Robert Pitz-Paal, High temperature solar concentrators (central receiver systems / parabolic dishes / solar furnaces), DLR,Institut für Technische Thermodynamik Solarforschung, Germany, Encyclopedia of Life Support Systems (EOLSS), http://www.eolss.net/outlinecomponents/Solar-EnergyConversion-Photoenergy-Systems.aspx; 